\title{
A Review of dental health issues in Parkinson`s disease patients
}

\author{
Borislav Ivanov ${ }^{1}$, Stefan Peev ${ }^{2}$, Mario Milkov ${ }^{3}$, Ara \\ Kaprelyan ${ }^{4}$, Ivan Dimitrov ${ }^{5}$.
}

1. Department of Clinical Medical Sciences, Faculty of Dental Medicine, Medical University, Varna, Bulgaria;

2. Department of Periodontology and Dental Implantology, Faculty of Dental Medicine, Medical University, Varna, Bulgaria;

3. Department of Prosthetic Dental Medicine and Orthodontics, Faculty of Dental Medicine, Medical University, Varna, Bulgaria;

4. Department of Neurological Diseases and Neurosciences, Faculty of Medicine, Medical University, Varna, Bulgaria;

5. Department of Healthcare, Sliven Affiliate, Medical University, Varna, Bulgaria;

\footnotetext{
Abstract

Parkinson's disease (PD) is the second most common neurodegenerative disease. Its prevalence is expected to double in the next 20 years due to the increase in life expectancy. The disease symptoms negatively impact patients' oral and dental health.

Dental specialists must be aware of PD clinical manifestations and meet effectively the challenges for office management and homecare.
} 
Keywords: Parkinson's disease, dental management, dental health issues

\section{Background}

Due to the increase in life expectancy the percentage of aged people in the total population is increasing rapidly and the prevalence of Parkinson's disease (PD) is expected to double over the next 20 years $(1,2$, $3)$.

PD significantly impacts all aspects of life, including oral health. Both motor and non-motor PD symptoms negatively influence patients' capability to maintain proper oral hygiene.

The oral health of PD patients is generally worse compared to the general population (4). Significantly higher frequency of untreated caries, periodontal diseases, and missing teeth is observed $(2,5,6,7,8,9)$.

Patients with mild PD have been reported by some authors to have less carious teeth $(10,11)$.

Nevertheless, orofacial and dental problems become more marked with progression of the disease (12).

\section{DEFINITION OF PARKINSON`S DISEASE}

PD is a chronic progressive multisystem neurodegenerative disease, with extrapyramidal, neuropsychiatric, dysautonomic and sensory symptoms.

PD is a proteinopathy (alfa-synucleinopathy), linked to the development of abnormal intraneuronal inclusions (Lewy bodies, Lewy neurites), developing in 6 neuropathological stages, with degeneration of different, especially vulnerable neuronal types (13).

The disease was first systematically described by the British physician James Parkinson in an 1817 report, named „An Essay on the Shaking Palsy”. Later, Jean- Martin Charcot proposed the disease to be renamed in honor of J. Parkinson (14).

PD affects up to $2 \%$ of the population aged over 65 years, with incidence of 4 to 20 new cases per 100,000 per year and prevalence estimated at 100 to 180 per 100,000 . The average age of symptoms onset is approximately 60 years $(15,16)$.

The cause of PD still remains unknown. Genetic and environmental causes and interaction between environmental risk factors and genetic susceptibility are suggested. The pathogenesis of PD has been associated with occupational chronic exposure to solvents and metals, known to affect the central nervous system, and a high prevalence of extrapyramidal symptoms has been found in dental technicians (17). Evidence now suggests that chronic neuroinflammation is consistently associated with the pathophysiology of Parkinson's disease. Also, recently, systemic inflammation has been proposed as one of the contributing factors for neurodegeneration. Some authors suggest a possible link between Parkinson's disease and periodontal disease (18).

\section{SYMPTOMS OF PARKINSON`S DISEASE}


PD is a progressive disease with a long premotor phase. The first symptoms, such as fatigue, depression, sleep problems, and constipation, are discrete, unspecific and seem unrelated. After years to decades the motor symptoms emerge. The so-called cardinal motor signs of PD include resting tremor, rigidity, bradikynesia and postural instability.

Tremor is the most apparent symptom. It is usually a 4 to $6 \mathrm{~Hz}$ rest tremor, consisting of "pill-rolling" of the fingers and pronation-supination of the hands. It may also affect the legs, lips, chin, jaw or tongue. Jaw tremor usually presents with "up and down" movement and less frequently with "side to side" movement. Tremor of the tongue and lips may interfere with biting, chewing and swallowing. As a consequence, attrition, orofacial pain, discomfort of the temporomandibular joint, cracked teeth, and biting of the tongue, cheeks and lips can be observed.

Rigidity of the muscles is a resistance to passive movements, caused by increased muscle tone. The rigidity can be smooth with the "lead pipe" phenomenon or ratchety, jerky, oscillating, with the "cogwheel” phenomenon. Rigidity affects all striated body muscles, including the orofacial ones.

Bradykinesia refers to slowness of movement, paucity of spontaneous movements and decreased movement amplitude. Micrographia (small handwriting), hypomimia (decreased facial expression), decreased blink rate, and hypophonia (soft speech) are all due to bradykinesia. Involvement of orofacial muscles is manifested by masked face (hypomimia), monotone and soft voice (hypophonia) and drooling of saliva due to decreased spontaneous swallowing (dysphagia).

Motor symptoms of PD are always asymmetrical, with one body side more severely affected than the other. In the early stages, the symptoms remain unilateral.

Postural instability, with impaired balance and loss of righting reflexes, is typical in the late stages of the disease, usually after 8 years or more. It responds poorly to treatment and is a common source of disability due to falls and traumatism $(2,19)$.

Another group of symptoms, the non-motor symptoms (NMS), may precede the motor ones by years or decades. NMS include neuropsychiatric symptoms like cognitive deficits, depression, anxiety and apathy, dysautonomic symptoms, including gastrointestinal (sialorrhea, dysphagia, gastroparesis, constipation, and defecatory dysfunction), urovesical (nicturia, polakyuria, urge incontinence, retention, delayed onset, and weak stream), cardiovascular (orthostatic hypotension, changes of blood pressure and heart rate, and regional circulation), thermoregulatory and sudomotor (hyperhydrosis, hypertermia, seborrhea, and heat intolerance), sexual and pupilary (decreased dark adaptation), sleep disorders, fatigue, pain, olfactory and visual dysfunction.

Depression occurs in up to $65 \%$ of PD patients and may precede the motor symptoms. Cognitive impairment can be seen early in PD with deficits in attention, concentration and visuospatial orientation. Over $20 \%$ of patients develop mild cognitive impairment (MCl) and dementia is observed in 15 to $55 \%$ of cases, usually late in the disease's course. Such patients tend to ignore many daily self-care procedures, including dental hygiene, and are more likely to miss dental appointments and less likely to report dental pain or other problems. 
Autonomic dysfunction affects virtually all patients as a part of the main neuropathological process and some symptoms, for example obstipation, usually precede the cardinal motor signs $(20,21)$.

The clinical picture of late PD is dominated by dyskinesias and motor fluctuations. These phenomena occur at least in half of the patients treated with levodopa within the first 5 years of therapy.

Dyskinesia manifests as involuntary, quick "dance-like" movements at the peak dose of the levodopa effect, affecting any body part. Involvement of the neck and face consists of jaw closing and opening, lip pursing and smacking and lateral or anterior flexion, or extension of the neck $(19,22)$.

Motor fluctuations are due to the depletion of levodopa efficacy. They manifest as lack of alleviation of tremor, rigidity and bradykinesia, and may include „on - off” phenomena, „wearing off” and even „no on” (19).

The most common oral manifestations of PD are dysphagia, salivary dysfunction and burning mouth syndrome.

Dysphagia is due to the main pathological process in PD and affects up to $75 \%$ of patients. It contributes to weight loss, fear while eating, withdrawal when eating and drinking among other people, difficulties taking PD medications, stress, worsening of anxiety and depression.

Dysphagia may also result in gastroesophageal reflux, causing perimylolysis.

With the progression of the disease, the risk of so-called "silent aspiration" increases. It leads to aspiration of small amounts of food, liquids, saliva or stomach contents and high prevalence of aspiration pneumonia is reported in patients with PD $(2,16,23)$.

Salivary dysfunction is common and is due to the autonomic dysfunction in PD and the use of anticholinergic medications. It can result in both excessive salivation (sialorrhea) and xerostomia.

Sialorrhea affects up to $78 \%$ of patients and is manifested by excessive drooling, which can cause dysphagia and vice versa. The saliva is described as being thick and rope-like, and the leakage may result in coughing and choking, frequent lip wiping, angular cheilitis and increased embarrassment in patients (2, $16,23,24,25)$.

Xerostomia affects approximately $55 \%$ of patients with PD and severely impacts oral health.

The tissues in the mouth become dry and painful. Ulcerations, oral discomfort and problems with denture retention appear. Patients are prone to cavities, tooth sensitivity, roots caries and periodontal diseases.

Burning mouth syndrome is associated with xerostomia, levodopa medication, deficiencies in vitamins or minerals, hormonal imbalances and poor oral hygiene. Vulnerable areas are the tongue, hard palate, lips and the alveolar ridge of edentulous individuals $(2,23)$.

PD patients also experience decreased appetite, impaired taste and olfaction, speech difficulties, all of these impacting general and oral health $(5,23)$.

\section{DENTAL MANAGEMENT}


The access of PD patients would be facilitated by a parking in the vicinity of the dental office, an elevator for wheelchairs, wide doorways, corridors and halls, because transportation and access are recognized as a significant barrier to receiving adequate healthcare (16).

Visits must be planned for early morning, when the patient's attention and cooperation are at their best. In case of marked bradikynesia, an early appointment will be challenging for the patient and the visits could be planned for mid-morning. Patients must be instructed to take their levodopa 60 to 90 minutes before the visit to benefit from the optimal effect of antiparkinsonian medication during the dental procedures.

Due to urinary incontinence, the arriving patient may need a bathroom stop. Thermoregulatory dysfunction is another dysautonomic symptom. The temperature in the dental office must be adjusted and a blanket may be provided to keep the patient's warmth.

Sitting in the dental chair may need assistance for stabilization and holding. Inclination of the dental chair at 45 degrees will avoid the risk of aspiration due to dysphagia and sialorrhea and the effects of orthostatic hypotension. PD patients may not tolerate a supine position.

Prior to starting any procedure, the dentist must obtain detailed medical history, contact the patient's healthcare team, determine the presence of comorbid illnesses and their medication (26). The dentist could use a patient's diary and should note the time and frequency of involuntary movements.

After a comprehensive examination, the current dental status and needs must be discussed with the patient and the caregiver. Informed consent must be obtained and a treatment plan must be devised. The dentist must ensure understanding of the benefits and risks of proposed interventions in the case of patients with cognitive deficits.

Series of several short and frequent visits will be more appropriate for PD patients. They usually do not tolerate long chair time, need shorter waiting time, their attention and cooperation exhaust and the medication effect depletes fast. The time frame of optimal medication response will decrease with the disease's progression. Visits should be planned every 3 or 4 months for adequate preventive care. In case of bedridden patients, home visits must be considered.

Patients with a mild and well-controlled disease do not pose dental management problems. Antibiotic prophylaxis is not usually needed. Bleeding is normal unless the patient is taking anticoagulants for comorbid diseases. Vital signs should be monitored on every visit.

Rigidity and bradikynesia cause the so-called mask-like face of PD patients. This lack of facial expression and speech problems may significantly disturb communication. The dentist should ask questions, requiring only a "yes" or "no" response, anticipate a slower response time and allow enough time for the patient to answer. The caregiver may also help interpreting the patient's speech. The dentist should pay attention to eye or limb movement during the procedure, suggestive of pain.

Appropriate pain management will minimize distress. Administration of anesthesia with epinephrine (1: $100000)$ is a good choice, if there are no comorbidity or medication interactions. 
In patients taking levodopa and entacapone administration of local anesthetics must be limited to three cartridges of $2 \%$ lidocaine with 1: 100000 epinephrine every half hour. Selegiline should not be combined with epinephrine or levonordefrin. Local anesthesia contributes to the patient's comfort, but the loss of sensation will exacerbate dysphagia.

In some patients dental procedures can only be performed under general anesthesia. The dentist should discuss this option with the patient, his family, healthcare team and caregiver. The patient should be warned that the recovery period may be prolonged.

Dental treatment should be started at the early stages, to prevent serious evolution and tooth loss. Invasive and restorative procedures should be completed in the initial stages of the disease to minimize risk and because PD patients do not tolerate longer visits, and have reduced cooperation with the disease's progression.

In some cases soft restraints and stabilization devices could be used on patients with PD to help contain tremor and provide support during manipulations. Cradling of the head can also help alleviate involuntary movements.

Severe jaw tremor and orobuccal dyskinesia can present serious difficulties in the management. Stress, pain and anticipation of procedures may exacerbate the tremor, making manipulations extremely complicated.

Mouth props and bite blocks could be used for problems with mouth opening and muscle rigidity. Use of powered scaling devices and air polishers must be avoided because of swallowing difficulties, choking and aspiration risk. Adequate fluid suction is imperative for safe and comfortable manipulations. In severe bruxism the reconstruction of occlusal surfaces should be kept as flat as possible to avoid breakage, because such patients present with fractured teeth and significant attrition (16).

At each prophylaxis visit, fluoride varnish should be applied, and fluoridated toothpastes or chlorhexidine gels may be prescribed.

After completion of the manipulations, the dental chair should be raised slowly to avoid symptoms of orthostatic hypotension. Patients may need some adjustment time in a sitting position.

They should rely on assistance while walking to and from the dental office, entering and exiting the building, and utilizing the restroom.

Patients with cognitive deficits should not be left alone throughout their dental visit (2).

\section{RECOMMENDATIONS FOR HOMECARE}

The patient should be explicitly instructed and continuously motivated for the maintenance of good oral hygiene.

PD patients must maintain a healthy diet for adequate nutrition. With the disease's progression they avoid foods that require chewing, such as vegetables and meat, which are rich of nutrients, and switch to sugar 
rich, soft and sticky snacks (4). They are easier to chew and swallow, but predispose to the development of „sweet teeth”, with risk for cavities, caries and periodontal diseases.

The dentist should recommend to patients to have six food intakes daily: four meals and two snacks. Snacks between the meals must be sugar free, because the frequency of sugar contact is more damaging for teeth than the amount of sugar. The patients may use nutritional and vitamin drinks, sugar free tea or coffee.

Motor symptoms may compromise brushing abilities. Recommendations must be given for brushing the teeth at least twice a day. Patients may clean part of the mouth in the morning and the other part in the afternoon.

Grip capabilities may be affected and it may be impossible for the arm to be lifted to the mouth. Increasing the surface and enlargement of handles will decrease the extra efforts. The dentist may recommend use of extended or enlarged toothbrush handles (for example a brush fixed into a tennis ball or a bike handlebar), or prescribe a large hand-held brush, a brush with soft bristles and smaller head, a brush with an angled head, a three-sided brush, an interdental brush, a proxabrush or a brush with surround bristles. In cases of disruption of the fine and/or repetitive motions, patients may use powered toothbrushes. Heavier devices, however, may compromise patient's abilities.

The dentist should recommend to the patients the so-called "one handed strategy"- use of the stronger side of the body.

Toothpaste dispenser pumps, ejecting fixed amounts and water irrigators with plaque revealing tablets may also be helpful.

Patients with inability to maintain proper self-care may need assistance. The caregiver may stand behind the patient for brushing his teeth or right-handed assistants could brush patient's left sided teeth from behind and right side teeth in front of him.

Topical stannous fluoride gel may be prescribed for daily application.

The patients must consider the amount of paste and water in their mouth, because spitting out can be difficult, due to the reduced oral muscles control and aspiration is a real risk.

Rigidity and bradikynesia significantly disrupt flossing, so patients may be advised to use a floss-holder, or they may need the caregiver to floss their teeth.

The specialist should remind the patient to clean the vestibule and the tongue with a washcloth.

Decreased coordination of tongue and throat muscles may affect rinsing. Oral rinses must be used with caution to avoid aspiration. Patients must use fluoride and chlorhexidine rinses. Alcohol-based mouthwashes are contraindicated. Dentists may recommend the use of a straw when rinsing or dipping the toothbrush in oral rinse.

III-fitting dentures and poor denture retention are another problem to consider, with significant impact on eating and social life. Changes of muscle tone and control, xerostomia or sialorrhea, weight loss, poorly designed, old or worn dentures are all factors for loose dentures. Ulceration and infection leads to discomfort, pain, infection, eating and speaking difficulties and contribute to a lack of oral hygiene selfcare. 
The dentist should advise the patient and the caregiver to clean dentures over a sink full of water using nailbrush and soap and water or denture pastes, and to avoid use of bleach or toothpaste on dentures. Dentures must not be left in hot solution for too long, to avoid damage of plastics. In case of discoloration, due to the contact with chewed or liquid levodopa, rinsing is appropriate after each medication dose. Persistent stains may also be removed by the dentist $(2,5,16,21,23,27)$. The best way to avoid problems with traditional dentures is the use of implant-supported denture or overdenture, which will allow for increased function and retention $(2,28,29)$.

Medical consultation is required for patients with difficulties placing and holding oral sleep apnea appliances. The use of continuous positive airway pressure (CPAP) device should be considered in such cases (27).

To diminish the risk of attrition and teeth breakage from falls, use of a mouth-guard may be most beneficial (2).

Patients with cognitive deficits may benefit from sticky notes at the brushing area to remind them the necessary steps. Caregivers can remind them to brush and floss as well. Dual tasks must be avoided.

The noise from powered toothbrushes and other devices may frighten patients with dementia, so their further use must be considered $(2,23)$.

In some cases temporomandibular joint problems develop, such as hypermobility of the mandible and frequent subluxation. Herbst appliances are an option, but because of rigidity and bradykinesia the patients may need assistance for placement $(27,30)$.

In case of gastroesophageal reflux, the use of calcium phosphate technologies and fluoride can lead to strengthening of the enamel.

Patients with xerostomia may benefit from frequent sips of water, sugar-free hard candies, and sugar-free, xylitol-containing gum, salivary substitutes made of carboxymethylcellulose, with viscosity similar to saliva, or fluoridated salivary substitutes. Cholinergic agonist drugs, pilocarpine and cevimeline $\mathrm{HCl}$, may also be prescribed.

Possible treatments of burning mouth syndrome include treating iron, vitamin B and other deficiencies, antifungal therapy, clonazepam, alpha-lipoic acid and cognitive behavioural therapy (2)..

\section{Conclusion}

PD symptoms negatively influence all aspects of a patient's life.

Different barriers hinder PD patients, as part of the elderly population, to receive appropriate healthcare. Dental specialists play an important role in maintaining optimal orodental health and the quality of life in $\mathrm{PD}$, providing adequate preventive and therapeutic strategies..

\section{References}


1. Alexander R, Gage T. Parkinson's disease: an update for dentists. Gen Dent. 2000, 48, 5, 572 580 .

2. De Bowes S, Tolle S, Bruhn A. Parkinson's disease: considerations for dental hygienists. Int J Dent Hyg. 2013, 11, 1, 15-21.

3. Nitschke I. Fundamentals of dentistry for geriatric rehabilitation - an introduction to geriatric dentistry. Z Gerontol Geriatr. 2000, 33 Suppl 1, 45-49.

4. Müller T, Palluch R, Jackowski J. Caries and periodontal disease in patients with Parkinson's disease. Spec Care Dentist. 2011, 31, 5, 178-181.

5. Cicciu M, Risitano G, Giudice G, Bramanti E. Periodontal Health and Caries Prevalence Evaluation in Patients Affected by Parkinson's Disease. Hindawi Publishing Corporation Parkinson's Disease Volume 2012, Article ID 541908, 6 pages doi: 10.1155/2012/541908.

6. Einarsdóttir E, Gunnsteinsdóttir H, Hallsdóttir M, Sveinsson S, Jónsdóttir S, Olafsson V, et al. Dental health of patients with Parkinson's disease in Iceland. Spec Care Dentist. 2009, 29, 3, 123127.

7. Katyayan P, Katyayan M, Nugala B. Dental management of Parkinson's disease: a case report. N Y State Dent J. 2013, 79, 5, 33-39.

8. Nakayama Y, Washio M, Mori M. Oral health conditions in patients with Parkinson's disease. J Epidemiol. 2004, 14, 5, 143-150.

9. Pradeep A, Singh S, Martande S, Raju A, Rustagi T, Suke D, Naik S. Clinical evaluation of the periodontal health condition and oral health awareness in Parkinson's disease patients. Gerodontology. 2015, 32, 2, 100-106.

10. Fukayo S. Dental status in outpatients with Parkinson's disease. Nihon Eiseigaku Zasshi. 2002, 57, 3, 585-590.

11. Fukayo S, Nonaka K, Shimizu T, Yano E. Oral health of patients with Parkinson's disease: factors related to their better dental status. Tohoku J Exp Med. 2003, 201, 3, 171-179.

12. Bakke M, Larsen S, Lautrup C, Karlsborg M. Orofacial function and oral health in patients with Parkinson's disease. Eur J Oral Sci. 2011, 119, 1, 27-32.

13. Braak H, Del Tredici K. Neuroanatomy and Pathology of Sporadic Parkinson's Disease. ISBN: 978-3-540-79849-1. 2009 Springer- Verlag Berlin Heidelberg. 119.

14. Lees A. Unresolved issues relating to the shaking palsy on the celebration of James Parkinson's 250th birthday. Mov. Disord. 2007, 22, Suppl 17, S327- 334.

15. Grosset D, Grosset K, Okun M, Fernandez H. Parkinson's Disease. 2009 Manson Publishing Ltd. ISBN: 978-1-84076-101-6.,176. 
16. Hodgson M, Norman M, Simmer-Beck M. Providing care to patients with Parkinson's disease. http://www.dimensionsofdentalhygiene.com/2014/03_March/Features/Providing_Care_to_Patients _with_Parkinsons_Disease.aspx.

17. Fabrizio E, Vanacore N, Valente M, Rubino A, Meco, G. High prevalence of extrapyramidal signs and symptoms in a group of Italian dental technicians. BMC Neurology 2007, 7, 24-28.

18. Kaur T, Uppoor A, Naik D. Parkinson's disease and periodontitis- the missing link? A review. Gerodontology. 2015 Feb 9. doi: 10.1111/ger.12188.

19. Browner N, Frucht S. Movement Disorders in Dental Practice. In: Lamster, I., Northridge, M. Improving Oral Health for the Elderly. An Interdisciplinary Approach. ISBN: 978-0-387-74336-3. 2008 Springer Science+Business Media, LLC, 506.

20. Ivanov B. Nonmotor symptoms in Parkinson's disease (in Bulgarian). ISBN 978-954-92624-52. Aktualna Medicina Varna Editions, 2012. 143.

21. Noble J. Dental Health and Parkinson's Disease. http://www.pdf.org/en/winter09_dental.

22. Ernest E. Hand Tremor with Dental Medicine Implications. 2009, Volume 9, Issue \#7. http://www.practicalpainmanagement.com/pain/maxillofacial/hand-tremor-dentalmedicineimplications.

23. Friedlander A, Mahler M, Norman K, Ettinger R. Parkinson disease: systemic and orofacial manifestations, medical and dental management. J Am Dent Assoc. 2009, 140, 6, 658-669.

24. Kalf J, de Swart B, Borm G, Bloem B, Munneke M. Prevalence and definition of drooling in Parkinson's disease: a systematic review. J Neurol 2009, 256, 1391-1396.

25. Srivanitchapoom P, Pandey S, Hallett M. Drooling in Parkinson's disease: a review. Parkinsonism Relat Disord. 2014, 20, 11, 1109-1118.

26. Johnson C, Shynett B, Johnson C, Maldonado B. Rapid onset of Parkinsonian- like symptoms in a geriatric dental patient: a case report. Compend Contin Educ Dent. 2006, 27, 11, 622- 625; quiz 626.

27. Burgess J. Management of the Dental Patient With Neurological Disease. 2013. http://emedicine.medscape.com/article/2091727-overview\#a1.

28. Haralur S. Clinical Strategies for Complete Denture Rehabilitation in a Patient with Parkinson Disease and Reduced Neuromuscular Control. Case Report. Hindawi Publishing Corporation. Case Reports in Dentistry. Volume 2015, Article ID 352878, 5 pages. http://dx.doi.org/10.1155/2015/352878.

29. Packer M, Nikitin V, Coward T, Davis D, Fiske J. The potential benefits of dental implants on the oral health quality of life of people with Parkinson's disease. Gerodontology. 2009, 26, 1, 1118. 
30. Silva P, Biasotto- Gonzalez D, Motta L, Silva S, Ferrari R, Fernandes K, et al. Impact in oral health and the prevalence of temporomandibular disorder in individuals with Parkinson's disease. J Phys Ther Sci. 2015, 27, 3, 887-891.

\section{Corresponding author:}

Borislav D. Ivanov, MD, PhD, Department of Clinical Medical Sciences

Faculty of Dental Medicine, Medical University, Varna, Bulgaria

84 "Tsar Osvoboditel" Str., Varna

GSM: 0889698416

E-mail: borislav.ivanov@mail.bg 